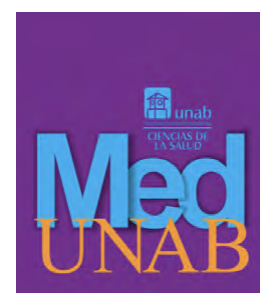

REVISTA DE LA FACULTAD

DE CIENCIAS DE LA SALUD

\title{
Reversión de anticoagulantes orales directos: una perspectiva desde Urgencias
}

Reversion of direct oral anticoagulants: an outlook from the Emergency Ward

Reversão de anticoagulantes orais diretos: uma perspectiva da Emergência

Mateo Zuluaga-Gómez, $M{ }^{1}{ }^{1}$ (iD, Nicolás Zuluaga-Arbeláez, $M d^{2}$ iD, Marie Claire Berrouet-Mejía, $\mathrm{Md}^{3}$ (iD, Andrés Felipe Estrada-Atehortúa, $M d^{4}{ }^{4}$ iD

1. Médico, Universidad Pontificia Bolivariana. Residente Medicina de Urgencias Universidad CES. Especialización Gerencia de IPS. Universidad CES. Docente cátedra Universidad Pontificia Bolivariana. Instructor CEMPAS, Universidad CES. Línea de Investigación Medicina de Urgencias y Toxicología - Grupo de investigación Especialidades Médicas y Quirúrgicas. Universidad CES. Medellín, Colombia.

2. Médico, Residente Médicina Interna. Universidad CES, Medellín, Colombia.

3. Médica Especialista en Toxicología Clínica Universidad de Antioquia, Toxicóloga Hospital General de Medellín - Clínica SOMA.Docente de farmacología y toxicología. Universidad CES. Línea de Investigación Medicina de Urgencias y Toxicología - Grupo de investigación Especialidades Médicas y Quirúrgicas. Universidad CES. Grupo de investigación HGM - CES. Medellín, Colombia.

4. Médico Especialista en Medicina de Urgencias, Universidad CES. Urgentólogo Hospital Pablo Tobón Uribe. Especialista en Gerencia de IPS Universidad CES. Docente cátedra Facultad de Medicina UPB. Docente adscrito Universidad CES. Línea de Investigación Medicina de Urgencias y Toxicología - Grupo de investigación Especialidades Médicas y Quirúrgicas. Universidad CES. Medellín, Colombia.

Correspondencia. Mateo Zuluaga-Gómez. Calle 28 Sur Número 43 A 50 Apartamento 1408 Edificio Ciudadela Santa Mónica, Envigado, Antioquia. Email.mateozg92@hotmail.com; zuluagag.mateo@uces.edu.co

\section{INFORMACIÓN DEL ARTÍCULO:}

Artículo recibido: 10 de Febrero del 2020

Artículo aceptado: 08 de Julio del 2020

DOI: https://doi.org/10.29375/01237047.3841

Cómo citar: Zuluaga-Gómez M, Zuluaga-Arbeláez N, Berrouet-Mejía MC, Estrada-Atehortua AF. Reversión de anticoagulantes orales directos: una perspectiva desde Urgencias. MedUNAB. 2020;23(3): 483-490. Doi: https:// doi.org/10.29375/01237047.3841

\section{RESUMEN}

Introducción. El sangrado en contexto de anticoagulación es uno de los riesgos que tienen ciertos pacientes durante determinados tratamientos, y es potencialmente mortal. Es importante conocer la farmacocinética de estas moléculas para predecir 
cuál será su comportamiento. Además se requiere juicio clínico en todos los casos de sangrado para determinar el manejo de acuerdo a la severidad. El objetivo de esta revisión es presentar un enfoque del paciente anticoagulado con sangrado en el servicio de urgencias. Temas tratados. Farmacocinética y farmacodinámica, generalidades, sangrado mayor y no mayor, opciones de reversión y tratamiento. Conclusión. En presencia de anticoagulantes orales directos (DOACS) se debe evaluar la gravedad del sangrado y el grado de anticoagulación, pues las estrategias de manejo se orientan dependiendo de si se trata de un sangrado mayor o menor.

Palabras claves:

Anticoagulantes directos; sangrado; idarucizumab; andexanet; Medicamentos Hemoderivados.

\section{ABSTRACT}

Introduction. Bleeding in the context of anticoagulation is one of the risks faced by some patients during certain treatments, and is potentially deadly. It is important to be aware of the pharmacokinetics of these molecules in order to predict their behavior. Clinical judgment is also required in all cases of bleeding, because management will depend on its severity. The purpose of this review is to present an approach to an anticoagulated patient with bleeding in the emergency ward. Topics Discussed. Pharmacokinetics and pharmacodynamics, generalities, major and nonmajor bleeding, reversal options and treatment. Conclusion. If direct oral anticoagulants (DOACS) are present, it is necessary to assess the severity of bleeding and the degree of anticoagulation, because the management treatment depends on whether bleeding is major or minor.

Keywords:

Direct anticoagulants; bleeding; idarucizumab; andexanet; Blood-Derivative Drugs.

\section{RESUMO}

Introdução. O sangramento no contexto da anticoagulação é um dos riscos que alguns pacientes apresentam durante certos tratamentos e é potencialmente fatal. É importante conhecer a farmacocinética dessas moléculas para prever qual será o seu comportamento. Além disso, o julgamento clínico é necessário em todos os casos de sangramento para determinar o manejo de acordo com a gravidade. O objetivo desta revisão é apresentar uma abordagem do paciente anticoagulado com sangramento no departamento de emergência. Tópicos abordados. Farmacocinética e farmacodinâmica, visão geral, sangramento maior e não maior, opções de reversão e tratamento. Conclusão. Na presença de anticoagulantes orais diretos (DOACS), a gravidade do sangramento e o grau de anticoagulação devem ser avaliados, uma vez que são orientadas as estratégias de manejo de acordo com o sangramento, se for maior ou menor

Palavras-chave:

Anticoagulantes diretos; sangramento; Idarucizumabe e Andexanet; Medicamentos Hemoderivados.

\section{Introducción}

Los anticoagulantes orales directos (DOAC) como el dabigatrán, rivaroxabán, apixabán o el edoxabán se han convertido en alternativas a los antagonistas de la Vitamina $\mathrm{K}$ y de las heparinas de bajo peso molecular en la prevención y tratamiento de varias afecciones cardiovasculares. Estos medicamentos han demostrado que son al menos tan efectivos y seguros como la terapia estándar en prevenciones de accidente cerebrovascular en pacientes con fibrilación auricular no valvular, así como de trombosis venosa profunda recurrente. También previenen -al igual que la estándar- el tromboembolismo después del reemplazo total de cadera y rodilla. Además son una alternativa al tratamiento de trombosis venosa profunda y tromboembolismo pulmonar (1).

En el mundo hay una tendencia hacia una mayor prescripción de DOAC. Para Inglaterra, por ejemplo, las prescripciones aumentaron del $9 \%$ en 2014 al $74 \%$ en 2019, mientras que la de warfarina disminuyó (2). En Colombia, aunque los DOAC no están cubiertos por el Plan Obligatorio de Salud (POS) son frecuentemente usados para las indicaciones mencionadas. $\mathrm{Su}$ popularidad tiene relación con la eficacia demostrada en diferentes ensayos clínicos y por varias de sus bondades como un efecto terapéutico más predecible, o que no 
requieren monitoreo del efecto anticoagulante y tienen menos interacciones con otros fármacos y alimentos.

La mayoría de los datos disponibles que evalúan los DOAC y el riesgo de sangrado han sido tomados de los ensayos en fibrilación auricular ARISTOTLE, RE-LY y ROCKET-AF, que en general mostraron menores tasas de hemorragia intracraneal y hemorragia mayor que el suministro de warfarina $(3,4)$. Sin embargo, el uso de cualquier anticoagulante se asocia con un mayor riesgo de sangrado y las complicaciones hemorrágicas pueden ser potencialmente mortales. Cuando esto ocurre es importante conocer la farmacocinética de estas moléculas para predecir cuál será su comportamiento. Además se requiere de un diagnóstico clínico en todos los casos de sangrado para determinar el riesgo y el manejo más adecuado. El objetivo de esta revisión es presentar el caso de un paciente con sangrado que fue anticoagulado en el servicio de Urgencias.

\section{Farmacocinética y farmacodinamia de los DOAC}

Los DOAC son medicamentos orales que inhiben directamente una enzima específica en la cascada de la coagulación. El dabigatrán inhibe directamente a la trombina (o factor activado II), mientras que el apixabán, rivaroxabán y el edoxabán inhiben al factor activado X. El estado de anticoagulación depende tanto del agente específico como de la cantidad suministrada, el tiempo transcurrido desde la última dosis, y las funciones renal y hepática. Es por eso que se deben conocer las particularidades de cada molécula:

- Dabigatrán etexilato: Es un profármaco administrado por vía oral que una vez en el hígado se convierte en dabigatrán. Su vida media es de 12 a 17 horas en individuos con función renal normal. La unión a proteínas es baja (de un 35\%) y se elimina por vía renal en un $80 \%(5,6)$.

- Rivaroxaban: Tiene una vida media de 5 a 9 horas. El 33\% de la molécula se excreta por vía renal sin metabolizarse. Su unión a proteínas plasmáticas es alta $(>90 \%)$. Es metabolizado en el hígado a través del citocromo CYP-3A4 (7).

- Apixaban: Tiene una vida media de 8 a 15 horas. Es un medicamento que se absorbe rápidamente por vía oral con una biodisponibilidad de casi el $50 \%$. Es metabolizado vía hepática por la enzima CYP-3A4. Se elimina el $25 \%$ en la orina y el $75 \%$ en las heces $(8,9)$.
- Edoxaban: Esta molécula, que aún no se encuentra disponible en Colombia, tiene una vida media de entre 6 y 11 horas. Presenta un perfil farmacocinético lineal. Tiene una biodisponibilidad oral del $62 \%$, pero a diferencia de otros inhibidores del factor activo $X$, se une poco a proteínas plasmáticas y no se biotransforma a través del citocromo CYP-3A4. Se elimina por vía biliar y renal (10).

\section{Enfoque del paciente con sangrado}

Guiar el manejo del paciente con sangrado en presencia de DOAC exige conocer la gravedad de la hemorragia, el estado de la anticoagulación y la indicación subyacente para el anticoagulante.

En la consulta se deben evaluar la magnitud y la localización del sangrado por medio de historia clínica y un examen físico, pues clasificar al paciente según el grado de sangrado ayuda a guiar el manejo: los pacientes con mayor hemorragia requieren de tratamientos más agresivos como estrategias de reversión, mientras que en la hemorragia menor se puede considerar sólo la observación y medidas locales (11), incluso sin suspender la anticoagulación, como se aprecia en el Esquema 1.

Para establecer criterios objetivos que ayuden a la investigación y al tratamiento, la Sociedad Internacional de Trombosis y Hemostasia ofrece una definición que debería ser aplicable a los estudios con todos los agentes que interfieren en la hemostasia. Así pues, el sangrado mayor se define como:

- Sangrado que lleva a la muerte.

- Sangrado sintomático en un área u órgano crítico, como intracraneal, intraespinal, intraocular, retroperitoneal, intra-articular, pericárdico o intramuscular con compartimento.

- Sangrado que causa una caída en el nivel de hemoglobina de $2 \mathrm{~g}$ o más, o que conduce a una transfusión de dos o más unidades de sangre completa o glóbulos rojos.

La hemorragia menor también es clínicamente significativa. Requiere de una evaluación de atención médica o de un tratamiento menos invasivo, como menorragia severa, equimosis o epistaxis (12). 
Esquema 1. Algoritmo de manejo en caso de sangrado por anticoagulantes orales directos.

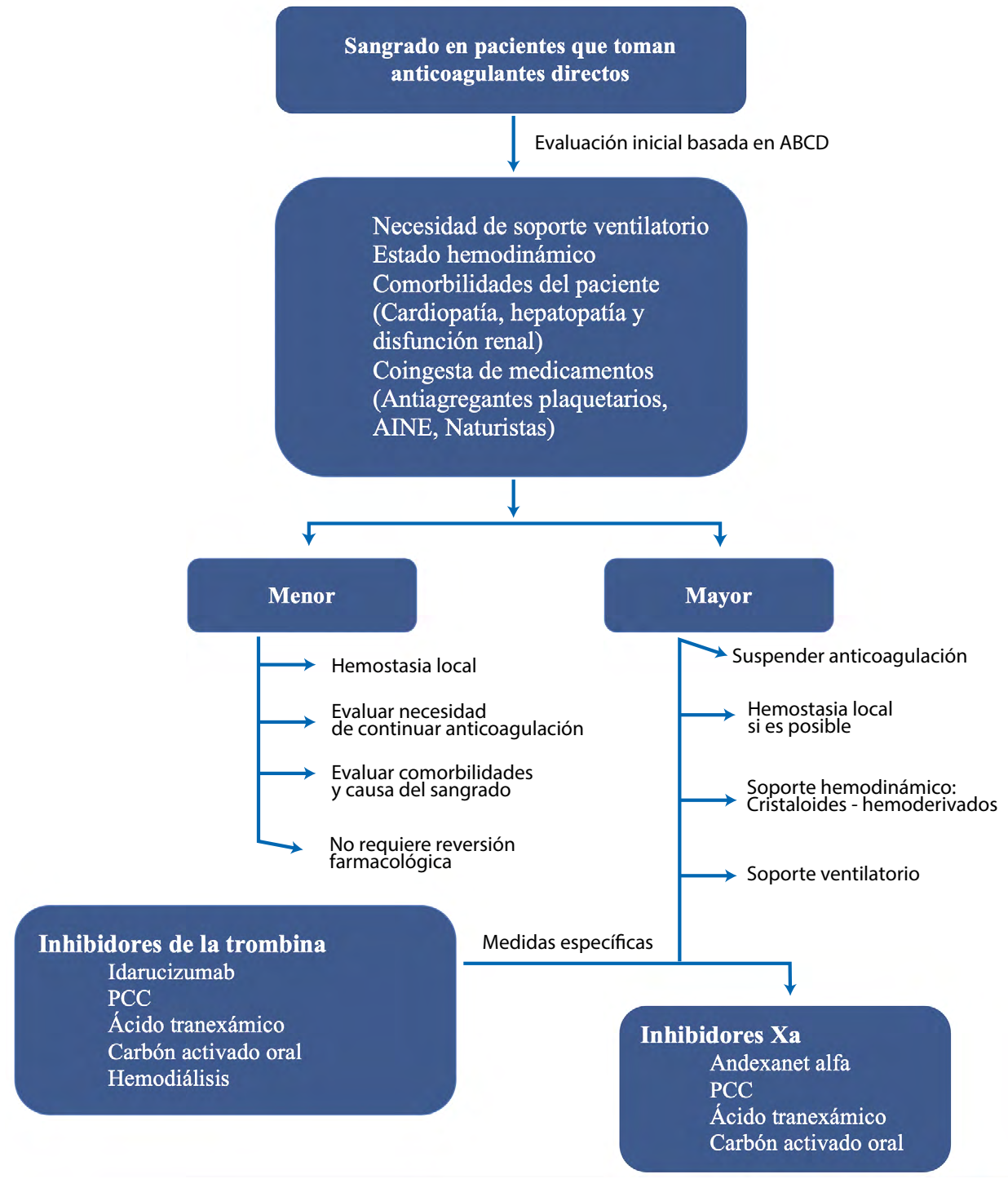

Fuente: elaboración propia.

Otro aspecto en la evaluación del paciente con sangrado es conocer el estado de anticoagulación, y en este aspecto, como ya se mencionó, es importante tener en cuenta las propiedades farmacocinéticas de cada molécula, pues se puede considerar que luego de la duración equivalente a cinco vidas medias de la molécula desde que se suministró la dosis, el paciente ya no está bajo el efecto de la anticoagulación. Además, la vida media depende de la función renal y hepática. Para los inhibidores del factor activo $\mathrm{X}$, la insuficiencia hepática grave podría provocar bioacumulación. Los pacientes con insuficiencia renal grave pueden tener un mayor grado o duración de la anticoagulación, dadas las características de excreción renal de cada molécula (11).
En el servicio de Urgencias, determinar el estado de anticoagulación por medio de las pruebas convencionales es limitado con los DOAC, pues las pruebas normales no pueden usarse como evidencia de que el efecto anticoagulante se ha resuelto o para eliminar la necesidad de intervenciones agresivas. Idealmente de acuerdo a la disponibilidad de los mismos, se recomienda evaluar el grado de anticoagulación mediante el tiempo de trombina diluido, el tiempo de ecarina y el ensayo cromogénico de ecarina en el caso del dabigatran o el ensayo cromogénico anti-Xa para el apixaban, edoxaban y rivaroxabán (13). La gran limitación es que estas pruebas no están ampliamente disponibles. 


\section{Manejo del paciente en el servicio de Urgencias}

Una vez se ha reconocido y clasificado el sangrado, el manejo del paciente se guiará de acuerdo a si es una hemorragia mayor o menor.

\subsection{Hemorragia mayor}

Se debe hacer un abordaje integral basado en una estrategia la $\mathrm{ABCD}$ de primeros auxilios: inicialmente valorar la vía aérea y el riesgo de deterioro de la misma; valorar principalmente el estado hemodinámico del paciente de manera rápida y continua; ofrecer medidas locales de control de la hemorragia en los casos de sangrado en sitios compresibles; suspender temporalmente el medicamento anticoagulante; posible transfusión de hemoderivados considerando si se requiere transfusión de glóbulos rojos, esto según el nivel de hemoglobina, tasa de sangrado y cantidad de pérdida de sangre. La transfusión de plaquetas solo se considera en casos de trombocitopenia; algunos expertos usan un umbral de transfusión $<50,000$ / microL, mientras que otros usan un recuento de plaquetas $<100,000 /$ microL, especialmente para hemorragias potencialmente mortales (11). El plasma fresco congelado puede administrarse como parte de un protocolo de transfusión masiva para reemplazar los factores de coagulación perdidos por el sangrado, pero no sirven como estrategia de reversión en el sangrado asociado a DOAC $(11,14)$.

Las anteriores son medidas generales de apoyo, pero las medidas específicas para cada molécula dependen de su mecanismo de acción:

\subsubsection{Inhibidores directos de la trombina como el dabigatrán}

En pacientes con hemorragia mayor o en quienes se requiere cirugía de urgencia es posible administrar idarucizumab, un fragmento de anticuerpo monoclonal humanizado que se fija al dabigatrán con afinidad muy alta. Es aproximadamente 300 veces más potente que la afinidad de fijación del dabigatrán a la trombina, de esa manera inactiva al dabigatrán y a sus metabolitos, neutralizando su efecto anticoagulante (15).

La eficacia de idarucizumab para revertir la anticoagulación con dabigatrán se demostró en el estudio RE-VERSE AD. Este fue un estudio de corte prospectivo que tuvo como objetivo evaluar la seguridad y la eficacia de la administración intravenosa de $5 \mathrm{~g}$ de idarucizumab en pacientes con un sangrado severo o que requerían un procedimiento urgente. Los resultados de un análisis intermedio de 90 pacientes en el estudio permitieron la aprobación para su uso por parte de la Administración de Drogas y Alimentos de los Estados Unidos (FDA), pues se demostró que el idarucizumab logró normalizar el tiempo directo de trombina en $98 \%$ de los pacientes con sangrado y en el $93 \%$ de los pacientes prequirúrgicos. En pacientes sometidos a procedimientos, la hemostasia era normal en el $92 \%$ de los casos. En pacientes con sangrado, el cese se logró dentro de un tiempo medio de 3.5 a 4.5 horas. La tasa de complicaciones trombóticas fue del 6\% (16).

La dosis recomendada es de 5 gramos vía intravenosa. La presentación del producto es de dos viales de 2.5 gramos en $25 \mathrm{ml}$ cada uno. No se deben administrar con más de 15 minutos de diferencia entre el primero y el segundo. Si el idarucizumab no está disponible, se sugiere la administración de un concentrado de complejo protrombínico, con productos derivados del plasma y que contiene los factores dependientes de vitamina $\mathrm{K}$; II, VII, IX y X de forma activa, como es el caso del FEIBA o de forma inactiva como el OCTAPLEX. La dosis del primero es de 50 a 80 unidades / $\mathrm{kg}$ y del segundo de 25 a 50 unidades $/ \mathrm{kg}$. No hay estudios controlados aleatorizados en humanos que demuestren la eficacia de anticoagulantes directos en pacientes con sangrado activo secundario (16).

En casos donde no hay disponibilidad de reversión farmacológica, hay evidencia débil de que la de hemodiálisis podría disminuir la concentración sérica de Dabigatrán, dada su baja unión a proteínas plasmáticas (17).

Medicamentos como el ácido tranexámico, por su bajo costo y bajo perfil de eventos adversos, se han recomendado por expertos en guías de manejo de pacientes anticoagulados con anticoagulantes directos. No hay estudios que respalden esta indicación. La dosis se extrapola del contexto de hemorragia intracraneana: 1 gramo Intravenoso durante 10 minutos seguido de 1 gramo durante 8 horas como infusión continua $(11,14)$.

También se puede usar carbón activado suministrado por vía oral para eliminar el fármaco no absorbido, si es que la última dosis fue durante las dos horas anteriores y el paciente puede tolerar la administración de carbón vía oral. 


\subsubsection{Inhibidores directos del factor activo $X$ como el apixaban, rivaroxaban, edoxaban}

En los pacientes con hemorragia mayor o cirugía urgente los complejos de concentrado de protrombina son una estrategia de reversión, esto basado en estudios in vitro en animales y grupos de voluntarios sanos a quienes se les suministran estos medicamentos. Ninguno de estos agentes ha demostrado eficacia o seguridad $(18,19)$.

En sujetos sanos que recibieron rivaroxabán, la infusión de PCC de 4 factores $(50 \mathrm{u} / \mathrm{kg})$ corrigió la prolongación del TP inducida por rivaroxabán y el potencial anormal de trombina endógena, pero no demostró corrección de las pruebas de coagulación para el dabigatrán. La PCC de cuatro factores y la PCC de 3 factores redujeron la PT media en 2.5-3.5 segundos y 0.6-1.0 segundos respectivamente, pero no tuvieron ningún efecto sobre el PTT o anti-X activo $(20,21)$.

No existen datos sobre la dosis adecuada para los casos de sangrado mayor. En un estudio aleatorizado de pacientes tratados con edoxaban sometidos a una biopsia por punción a quienes se les aplicó una infusión de PCC de 4 factores o placebo. A la dosis más alta de PCC de 4 factores probados $(50 \mathrm{u} / \mathrm{kg})$, se observó una reversión estadísticamente significativa del efecto del edoxabán sobre la duración del sangrado (22).

El andexanet fue aprobado por la FDA en mayo de 2018 para la reversión de la anticoagulación por rivaroxabán y apixabán en individuos con hemorragias potencialmente mortales o no controladas. Es un factor activo $\mathrm{X}$ recombinante sin actividad enzimática y con la capacidad de unión a los inhibidores circulantes del factor activo X. Fue evaluado en un estudio multicéntrico, prospectivo y abierto de grupo único en el que se incluyeron 352 pacientes con hemorragia grave aguda a las 18 horas posteriores a la administración de rivaroxaban o apixaban. La hemostasis excelente o buena ocurrió en 204 de los 249 pacientes evaluados (el $82 \%$ ). En 30 días, la muerte ocurrió en 49 (el 14\%) y un evento trombótico en $34(10 \%)$.

No hay pruebas suficientes sobre los riesgos y los beneficios para favorecer más al PCC de 4 factores o al andexanet, pues no hay estudios que los comparen.

Dentro de otras medidas para controlar el sangrado se encuentran el uso de ácido tranexámico, con las mismas condiciones mencionadas para el dabigatrán, pues no hay evidencia fuerte para su uso. Si la última dosis del anticoagulante fue reciente, lo suficiente como para que el fármaco no se haya absorbido - por ejemplo, ocho horas para rivaroxaban, seis horas para apixaban o dos horas para edoxaban-, se podría administrar carbón activado. Los inhibidores orales directos del factor Xa disponibles no pueden dializarse, ya que están altamente unidos a proteínas.

En Colombia ya se dispone de idarucizumab, pero no se dispone aún de andexanet.

\subsection{Hemorragia menor}

El sangrado menor generalmente se puede manejar de manera conservadora utilizando medidas hemostáticas locales. La decisión de suspender la anticoagulación debe tomarse cuando al paciente se le va a realizar un procedimiento invasivo, cuando hay una alteración de la función renal o la hepática que aumente la probabilidad de progresión a una hemorragia más grave, o cuando existe preocupación por un sangrado lento en un sitio crítico.

En casos de hemorragia menor no se administra concentrados de complejo de protrombina o antídotos específicos.

\section{Conclusión}

La prescripción de los anticoagulantes orales directos ha aumentado con el tiempo porque ofrecen ciertas ventajas en comparación con la warfarina o las heparinas de bajo peso molecular. Sin embargo, aún existe un riesgo de sangrado y esta complicación puede ser potencialmente mortal. Cuando el paciente se presenta a urgencias con sangrado en presencia de DOAC se debe evaluar la gravedad del sangrado y el grado de anticoagulación, pues las estrategias de manejo se orientan dependiendo de si se trata de un sangrado mayor o menor.

\section{Referencias}

1. Chen A, Stecker E, A Warden B. Direct Oral Anticoagulant Use: A Practical Guide to Common Clinical Challenges. J Am Heart Assoc [Internet]. 2020 Jun 15 [citado 2020 Jun 27];e017559. Disponible en: http://www.ncbi.nlm.nih.gov/pubmed/32538234

2. Ho KH, Van Hove M, Leng G. Trends in anticoagulant prescribing: A review of local policies in English primary care [Internet]. Vol. 20, BMC Health Services Research. BioMed Central Ltd.; 2020 [citado 2020 Jun 27]. p. 279. https://doi.org/10.1186/s12913-020$\underline{5058-1}$ 
3. Ruff CT, Giugliano RP, Braunwald E, Hoffman EB, Deenadayalu N, Ezekowitz MD, et al. Comparison of the efficacy and safety of new oral anticoagulants with warfarin in patients with atrial fibrillation: A meta-analysis of randomised trials. Lancet [Internet]. 2014 [citado 2020 Jun 27];383(9921):955-62. https:// doi.org/10.1016/S0140-6736(13)62343-0

4. Providência R, Grove EL, Husted S, Barra S, Boveda $\mathrm{S}$, Morais J. A meta-analysis of phase III randomized controlled trials with novel oral anticoagulants in atrial fibrillation: Comparisons between direct thrombin inhibitors vs. factor $\mathrm{Xa}$ inhibitors and different dosing regimens. Thromb Res [Internet]. 2014 Dec 1 [citado 2020 Jun 28];134(6):1253-64. https://doi. org/10.1016/j.thromres.2014.10.002

5. Blech S, Ebner T, Ludwig-Schwellinger E, Stangier J, Roth W. The metabolism and disposition of the oral direct thrombin inhibitor, dabigatran, in humans. Drug Metab Dispos [Internet]. 2008 Feb [citado 2020 Jun 28];36(2):386-99. https://doi.org/10.1124/ dmd.107.019083

6. Stangier J. Clinical pharmacokinetics and pharmacodynamics of the oral direct thrombin inhibitor dabigatran etexilate [Internet]. Vol. 47, Clinical Pharmacokinetics. Clin Pharmacokinet; 2008 [citado 2020 Jun 28]. p. 285-95. https://doi. org/10.2165/00003088-200847050-00001

7. Eriksson BI, Quinlan DJ, Weitz JI. Comparative pharmacodynamics and pharmacokinetics of oral direct thrombin and factor Xa inhibitors in development [Internet]. Vol. 48, Clinical Pharmacokinetics. Clin Pharmacokinet; 2009 [citado 2020 Jun 28]. p. 1-22. https://doi.org/10.2165/0003088-200948010-00001

8. Cui Y, Song Y, Wang J, Yu Z, Schuster A, Barrett YC, et al. Single- and multiple-dose pharmacokinetics, pharmacodynamics, and safety of apixaban in healthy Chinese subjects. Clin Pharmacol Adv Appl [Internet]. 2013 Dec 6 [citado 2020 Jun 28];5:177-84. Disponible en:/pmc/articles/PMC3861362/?report=abstract

9. He K, Luettgen JM, Zhang D, He B, Grace JE, Xin $\mathrm{B}$, et al. Preclinical pharmacokinetics and pharmacodynamics of apixaban, a potent and selective factor Xa inhibitor. Eur J Drug Metab Pharmacokinet [Internet]. 2011 Sep [citado 2020 Jun 28];36(3):12939. https://doi.org/10.1007/s13318-011-0037-x

10. Tamargo J. Edoxabán. Propiedades farmacocinéticas y farmacodinámicas. Rev Esp Cardiol Supl [Internet]. 2016 Jan 1 [citado 2020 Jun 26];16:60-6. https://doi. org/10.1016/S1131-3587(16)30017-6

11. Tomaselli GF, Mahaffey KW, Cuker A, Dobesh PP, Doherty JU, Eikelboom JW, et al. 2017 ACC Expert Consensus Decision Pathway on Management of Bleeding in Patients on Oral Anticoagulants: A Report of the American College of Cardiology Task Force on Expert Consensus Decision Pathways. J Am
Coll Cardiol [Internet]. 2017 Dec 19 [citado 2020 Jun 28];70(24):3042-67. https://doi.org/10.1016/j. jacc.2017.09.1085

12. Schulman S, Kearon C. Definition of major bleeding in clinical investigations of antihemostatic medicinal products in non-surgical patients [Internet]. Vol. 3, Journal of Thrombosis and Haemostasis. J Thromb Haemost; 2005 [citado 2020 Jun 27]. p. 692-4. https:// doi.org/10.1111/j.1538-7836.2005.01204.x

13. Samuelson BT, Cuker A, Siegal DM, Crowther M, Garcia DA. Laboratory Assessment of the Anticoagulant Activity of Direct Oral Anticoagulants: A Systematic Review. Chest [Internet]. 2017 Jan 1 [citado 2020 Jun 27];151(1):127-38. https://doi. org/10.1016/j.chest.2016.08.1462

14. Shih AW, Crowther MA. Reversal of direct oral anticoagulants: A practical approach. Hematology [Internet]. 2016 [citado 2020 Jun 28];2016(1):612-9. https://doi.org/10.1182/asheducation-2016.1.612

15. Syed YY. Idarucizumab: A review as a reversal agent for dabigatran [Internet]. Vol. 16, American Journal of Cardiovascular Drugs. Springer International Publishing; 2016 [citado 2020 Jun 28]. p. 297-304. https://doi.org/10.1007/s40256-016-0181-4

16. Pollack C V., Reilly PA, Eikelboom J, Glund S, Verhamme P, Bernstein RA, et al. Idarucizumab for Dabigatran Reversal. N Engl J Med [Internet]. 2015 Aug 6 [citado 2020 Jun 28];373(6):511-20. https://doi. org/10.1056/NEJMoa1502000

17. Khadzhynov D, Wagner F, Formella S, Wiegert E, Moschetti V, Slowinski T, et al. Effective elimination of dabigatran by haemodialysis: A phase I singlecentre study in patients with end-stage renal disease. Thromb Haemost [Internet]. 2013 Feb 7 [citado 2020 Jun 28];109(4):596-605. https://doi.org/10.1160/ TH12-08-0573

18. Siegal DM. Managing target-specific oral anticoagulant associated bleeding including an update on pharmacological reversal agents. J Thromb Thrombolysis [Internet]. 2015 [citado 2020 Jun 28];39(3):395-402. https://doi.org/10.1007/s11239015-1167-9

19. Crowther M, Crowther MA. Antidotes for novel oral anticoagulants: Current status and future potential. Arterioscler Thromb Vasc Biol [Internet]. 2015 agosto 25 [citado 2020 Jun 28];35(8):1736-45. https://doi. org/10.1161/ATVBAHA.114.303402

20. Eerenberg ES, Kamphuisen PW, Sijpkens MK, Meijers JC, Buller HR, Levi M. Reversal of rivaroxaban and dabigatran by prothrombin complex concentrate: A randomized, placebo-controlled, crossover study in healthy subjects. Circulation [Internet]. 2011 Oct 4 [citado 2020 Jun 28];124(14):1573-9. https://doi. org/10.1161/CIRCULATIONAHA.111.029017 
21. M L, KT M, CF C, D K, SD B, SZ G, et al. Comparison of Three-Factor and Four-Factor Prothrombin Complex Concentrates Regarding Reversal of the Anticoagulant Effects of Rivaroxaban in Healthy Volunteers. J Thromb Haemost [Internet]. 2014 [citado 2020 Jun 28];12(9). https://doi.org/10.1111/jth.12599

22. Zahir H, Brown KS, Vandell AG, Desai M, Maa JF, Dishy V, et al. Edoxaban effects on bleeding following punch biopsy and reversal by a 4-factor prothrombin complex concentrate. Circulation [Internet]. 2015 [citado 2020 Jun 28];131(1):82-90. https://doi. org/10.1161/CIRCULATIONAHA.114.013445 